\title{
Continued professional development of teachers to facilitate language used in numeracy and mathematics
}

\author{
A-M Wium, B Louw \\ Department Speech-Language Pathology and Audiology, University of Limpopo \\ Anna-Marie Wium
}

Department Communication Pathology, University of Pretoria

Brenda Louw

Corresponding author: A Wium (anna-marie1.wium@ul.ac.za)

\begin{abstract}
Learners in South African schools have been found to perform poorly in mathematics because they do not understand the language used in solving mathematical problems. In order to improve academic performance teachers need to be made aware of the importance of language in the development of numeracy. A continued professional development (CPD) programme addressed this need. The purpose of the research was to understand how the participants implemented the strategies developed during the programme and how they perceived the support provided by the programme. The research was conducted over 2 years in semi-rural and urban contexts. As part of a more comprehensive mixed method study, the qualitative data referred to in this article were obtained through open-ended questions in questionnaires, focus groups, reflections in portfolios, and a research diary. Results showed that numeracy terminology was often used by learners that differed from standard terminology prescribed by the curriculum. The participants themselves did not necessarily understand the numeracy terminology and thus found it a challenge to implement curriculum outcomes. Issues related to language use of the participants in teaching numeracy were associated with the lack of resources available in the language of learning and teaching (LoLT). Some of the participants taught numeracy in English, rather than LoLT. The results indicated low teacher expectations of the learners. The CPD programme was considered valuable and effective. SLPs in schools need to be expand their role to provide CPD opportunities for teachers.
\end{abstract}

Keywords: teacher support, numeracy, collaboration, language, foundation phase, speech-language pathologist

S Afr J CD 2012;59(1):8-15. DOI:10.7196/SAJCD.121

\begin{abstract}
Background and contextualisation of the study It is widely acknowledged that learners in South Africa have performed poorly in numeracy benchmark tests, which was partly ascribed to inadequate language capabilities as many learners did not understand what was expected of them when they were assessed (Department of Basic Education, 2011). Such findings indicate a need to raise the level of learner achievement. However, raising learners' performance levels in the current context is complex as few learners from low socio-economic schools (SES) receive any preschool education, which impacts on their school readiness when they enter formal education (Botha, Maree \& de Witt, 2005). Furthermore, many of the teachers currently in the system received their training under a previous dispensation when education was fragmented and inequitable, resulting in inadequate professional training (Thusi, 2006). In addition, the new democracy brought about positive sociopolitical change, including a new national education curriculum. However, the implementation of a new curriculum requires additional support of teachers, which could be provided with continued professional development (CPD) activities.
\end{abstract}

This paper continues the reporting of the results of a body of research on the development of a CPD programme for foundation-phase teachers to facilitate the development of literacy skills, as well as the language used for the teaching of numeracy in the classroom (Wium, Louw, \& Eloff, 2010). Based on the premise that language is the key to all learning, this article focuses on how foundation-phase teachers can be supported to facilitate learners' language for numeracy and mathematics. The aim of the study was to develop a support programme for foundation-phase teachers to facilitate language for numeracy skills by using a three-pronged approach (Appendix A, Table A1), consisting of a training component (workshop), a practical component (classroom implementation of strategies), and a mentoring component (feedback by facilitator on lesson planning and portfolio assignments). The research objectives reported on in this article are firstly to determine how the participants implemented strategies that were acquired in a CPD programme to facilitate the language for numeracy in their classrooms, and secondly, to determine their perceptions of the support provided by the CPD programme.

\section{Numeracy, mathematics and language}

Numeracy is the ability to understand and reason with number, to act wisely in daily living activities, and to participate in civic life. It also allows one to use mathematics in order to solve problems. Learners therefore need both numeracy and mathematics for mathematical learning. The current South African curriculum includes numeracy in the foundation phase as basis for mathematics, which is presented in Gr 4 and onwards. It is therefore imperative that all children should acquire numeracy skills from early on.

According to Schleppegrell (2007: 140) 'Learning the language of a new discipline is part of learning the new discipline; in fact, the language and learning cannot be separated.' The language of mathematics needs to be acquired, since the teaching of mathematics occurs through discourses; students' work is assessed through oral and written communication, and learners process their ideas to develop understanding of numeracy and mathematics through language (Raiker, 2002). There are contrasting viewpoints on the relationship between language acquisition and the acquisition of numerical concepts. One group of researchers contends that there is a causal relationship between language acquisition and the acquisition of numerical concepts, while another viewpoint holds that by learning a conventional spoken language for number, children learn to reason numerically (Arvedson, 2002). Although the exact relationship remains complex and controversial, language competency plays an important role in the development of numeracy and mathematical skills. Language, mathematics and numerical cognition are all closely interrelated.

The role of language and social interaction in the learning of mathematical concepts is based on interactions between the teacher and learners, as 
well as within peer groups (Barwell, Leungh, Morgan \& Street, 2005). Teachers, as mediators and facilitators of learning, play a significant role in guiding discourses in the classroom, and also provide the necessary scaffolding through instruction, modelling and group-based activities in the use of language for reasoning (Mercer \& Sams, 2006).

\section{Learning theory in relation to the development of numeracy skills}

The developmentally appropriate practice (DAP) that was grounded in Piaget's stage-based theory on child development has now been replaced by a neo-Vygotskian sociocultural perspective. The sociocultural theory, together with the closely related activity theory', account for learning and development as mediated processes (Mercer \& Sams, 2006). The latter view considers social involvement in problem-solving activities as a crucial factor for individual development. Social experiences with more 'knowledgeable others' (e.g. parent or teacher) enable the child to develop understanding that facilitates further learning. Language is considered as both a psychological and cultural tool.

Learning first appears on a social plane (between people), and then on a psychological plane, which is within the child (also referred to as 'internalisation'). Early literacy and numeracy do not simply happen, but evolve through a social process that is based on children's relationships with significant others in their environment (e.g. parents, siblings, grandparents, teachers, extended family, etc.). Based on sociocultural theories, there is a close relationship between symbolic thinking and early literacy and numeracy. Communication and cognitive learning take place through informal socialisation, and without direct instruction. Learners with age-appropriate language proficiency acquire the language of mathematics as one component of a complex symbolic communication function.

Young children acquire mathematical concepts during play, and at the onset of Gr R typically developing children have acquired the emergent numeracy concepts (Torbeyns, van den Noortgate, \& Ghesquirer, 2002), as well as language of measurement and position in space, the selection criteria for sorting, exploring, building, and matching with shapes. The vocabulary that develops from this emergent phase of numeracy is of particular importance. A summary of emergent numeracy skills and the associated vocabulary are summarised in Table 1.

\section{Approaches to teaching of language for numeracy and mathematics}

The issue of how vocabulary for numeracy should be taught is debated from opposing perspectives. Vacca and Vacca (1996) conclude

\section{Table 1. Emergent numeracy skills with required matching vocabulary (based on Torbeyns et al., 2002)}

\begin{tabular}{ll}
\hline Concept & Vocabulary \\
\hline $\begin{array}{l}\text { Concept of comparison: } \\
\text { ability to compare objects } \\
\text { in terms of quantitative and } \\
\text { qualitative properties }\end{array}$ & $\begin{array}{l}\text { Same/different } \\
\text { More than/less than } \\
\text { Number words: one, two, three, four, etc. } \\
\text { Opposites: smallest/biggest; longest/shortest, } \\
\text { tallest/shortest, lots, many/few; most/least; } \\
\text { the same (equal) }\end{array}$ \\
$\begin{array}{l}\text { Classification: the } \\
\text { prerequisite is that learners } \\
\text { must be able to sort }\end{array}$ & $\begin{array}{l}\text { Comparative words, e.g. same/different; long/ } \\
\text { short; more/less; too many/not enough; none }\end{array}$ \\
$\begin{array}{l}\text { One-to-one correspondence } \\
\text { Also includes comparative words, e.g. same/ } \\
\text { different; long/short; more/less; too many/not } \\
\text { enough; none; degrees of comparison (e.g. } \\
\text { short, shorter, shortest) }\end{array}$ \\
Counting, plus all of the above \\
$\begin{array}{l}\text { General understanding } \\
\text { of numbers }\end{array}$
\end{tabular}

that the vocabulary of mathematics instruction should be taught directly. In contrast, Thompson and Rubenstein (2000) propose an enculturation with the language of mathematics, especially at the preschool level, which concurs with views on literacy development. Elkind (2001), however, states that neither the direct nor the indirect manner of instruction provides an answer, and suggests that children should learn mathematical vocabulary in both a formal and informal manner. This debate highlights the focus on the role of language and social interaction in the learning of numeracy and mathematics.

\section{Discourses used in classrooms}

The discourses used in classrooms are a determining factor in the acquisition of numeracy and mathematics. Figure 1 illustrates that the language for numeracy and mathematics requires competence on four different levels (Gawned, 1993). The developed CPD programme specifically addressed levels 3 and 4 of the numeracy discourses as these were more specific in terms of vocabulary and terminology used in the numeracy curriculum (refer to Table C1 in Appendix C).

\section{Language required for numeracy: Level 3}

With reference to Level 3 in Figure 1, four different numeracy discourses need to be considered, namely the language of reasoning (problem solving), the language of the numeracy or mathematics curriculum, the language of activities, and the language of mathematics literacy (Gawned, 1993). These domains of language use relate to the cognitive academic language proficiency (CALP) required in the numeracy skill area, which can only develop once competence in basic interactive communicative skills (BICS) have been achieved.

Learners have to acquire the terminology and vocabulary included in the subject material because it will allow them to communicate their thinking and will increase their confidence (Monroe \& Omre, 2002). Studies by Reeves and Long (1998) conducted in the Western Cape and by Setati (1999) with Gr 4 learners in Mamelodi, Gauteng, reported that incorrect use of mathematical language in classrooms had a negative effect on learning. Teachers need to be aware that the language they use to teach and to discuss numeracy also warrants careful consideration. Formal language in itself consists of procedural, calculative, and conceptual language that provides the reasons for proceeding or calculating in particular ways (refer to Appendix B, Table B1). Setati (1999) found lessons to be dominated by procedural discourse and that conceptual discourse was limited. Before teachers can affect any changes in practice they need to be cognisant of their own use of language and, if necessary, make purposeful modifications.

Gawned's (1993) description of the discourses used in mathematics classrooms remains relevant today and is also applicable to the language used in foundation-phase classrooms where a numeracy curriculum is

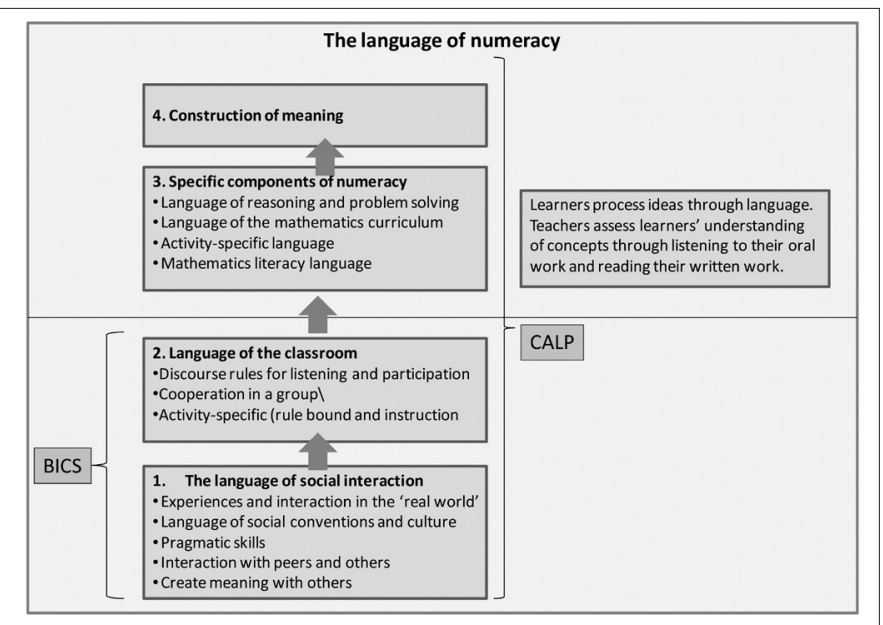

Fig. 1. The numeracy discourses used in classrooms (Gawned, 1993) (BICS = basic interactive communicative skills; CALP = cognitive academic language proficiency). 
followed (e.g. in the current context). The language used in mathematics builds on the language used in the numeracy curriculum and such learning therefore occurs on a continuum.

\section{The role of speech-language pathologists (SLPs) in supporting numeracy}

Children with early speech and language impairments are at risk for literacy and numeracy problems (Arvedson, 2002; Harrison, McLeod, Berthelsen \& Walker, 2009). Traditionally SLPs have provided direct services to children with speech and language impairment who experience literacy difficulties in schools. However, the role of the SLP in South Africa has recently evolved and expanded to provide support to teachers in literacy development of all learners (Kathard et al., 2011) because of challenges such as children having a different first language (L1) to the language of learning and teaching (LoLT), large classes, lack of resources and limited teacher training (Wium et al., 2010).

Unfortunately teachers may be unaware of the important role that language plays in numeracy and mathematics and as a result may not realise that SLPs can collaborate and support them in the teaching of early numeracy and mathematics. SLPs are well equipped to support teachers as they have knowledge of collaborative practice, current theories and developmental sequences of language development and use, as well as literacy and cognitive development, which includes numerical cognition. SLPs therefore need to become involved in the support of language for the development of numeracy, which calls for specific approaches to teaching and learning. In addition, SLPs are also required to take up leadership roles, such as providing professional development opportunities (ASHA, 2010).

Although the role of the SLP in numeracy and mathematics is recognised (Brennan, 2008; Harrison et al., 2009; Samuelson, 2008), limited research has been conducted on speech-language interventions targeting mathematical skills with children with speech and language impairment (Koponen, Mononen, Rasanen \& Ahonen, 2006). There is also a dearth of research on the role and involvement of SLPs in providing teacher support to facilitate the development of numeracy and mathematical skills in all learners. The aim of this article is to report on a CPD programme that focuses on teacher support regarding the language required for numeracy. The objectives were to determine how the participants implemented the strategies to facilitate the language for numeracy in their classrooms, and how they perceived the CPD programme.

\section{Methodology}

The qualitative research reported in this article is part of a more comprehensive mixed methods study (Wium et al., 2010), and the workshop and data focused on in this article were part of a larger data set.

\section{Participants}

\section{Selection criteria}

All participants included in this study were required to meet the following criteria:

- Be appointed in teaching positions in the foundation phase (Gr R, 1,2 and 3) at schools in the targeted schools of the two contexts. The Gauteng Department of Education (GDE) specified two particular districts and specific schools for this CPD programme and therefore only teachers from the assigned schools could be included.

- Be proficient in English as the training and procedures were developed in English.

- Participate voluntarily and not as a result of coercion by their superiors.

\section{Selection procedure}

The GDE assigned 24 low socio-economic schools (SES) in the Tshwane region to this project over a period of 2 consecutive years. There were
12 schools included from a semi-rural area, and 12 schools from an urban/densely populated area (including township schools and schools from informal settlements). Through a process of stratified sampling each school was required to allocate one teacher from each grade in the foundation phase, provided their participation was voluntary. With 48 teachers participating per annum the entire sample consisted of 96 teachers, which was considered sufficient to serve the purpose of this specific study.

The sample was fairly homogeneous in terms of contexts, grade levels represented, and the teachers' experience in teaching (but not in terms of qualification), and therefore is considered to be a realistic crosssection of the population. All the participants in the semi-rural context were female, whereas two of the urban participants were male.

A focus group consisting of 12 participants was conducted in each of the two contexts. With a total of 24 participants in the focus groups the sample was considered as representative (25\%) of the entire group that was trained, and allowed for attrition.

The research made use of the entire sample $(N=96)$ to complete the open-ended questionnaires, but also used a nested sample design for the selection of the participants in the focus group. The participants who volunteered to participate in the focus groups $(n=24)$ originated from the entire sample and therefore were similar to those in the rest of the study.

\section{Data collection methods and instruments}

A specific workshop on the topic 'Language in numeracy' was conducted in each context (refer to Appendix A, Table A1 for an outline of the workshop as described in the 'Training component'). In order to meet the specific objectives of this study qualitative data were collected through open-ended questions in questionnaires, narrative data obtained from a focus group with field notes and reflections in portfolios, as well as reflections of the researcher in a research diary. The participants reported on their perceived value of the $\mathrm{CPD}$, while the researchers made conclusive inferences by reflecting on the entire process of support provided. The two open-ended questions included in the questionnaires were related to the participants' perceptions of the workshop and their recommendations for change. Questionnaires were completed prior to and directly after training, and were placed in a box when participants exited the training venue for a tea break or at the end of the day.

A focus group discussion was conducted 3 - 4 weeks after the workshop in each of the two contexts. In the semi-rural context the focus group was conducted in a teachers' training centre, and in the urban context it was conducted in a conference room at the Department Communication Pathology, University of Pretoria because it was half-way between the townships. Each focus group was conducted in English, but participants were encouraged to respond in their language of preference should they feel the need. The district facilitators served as interpreters and/or translators for those who chose to use an indigenous language. Questions often had to be rephrased and repeated to accommodate participants with limited English language proficiency. The session was concluded with a summary of the meeting being read to the participants for approval ('member checking'). The content and construct validity of the focus group schedule were increased by following literature guidelines and having the questions reviewed by two experts in the field prior to use.

During the period of classroom implementation following the workshop, the participants were required to compile a portfolio consisting of their lesson planning for each week of the 3-week implementation period, their reflections on the strategies used, and some artefacts used in their teaching. The researcher continually reflected on the process of teacher support in a research diary. Entries to the research diary were made 
within hours of an event or contact with the participants and these reflections were shared with experts and colleagues, which elicited meta-reflection.

\section{Credibility}

Credibility was determined by randomly selecting $10 \%$ of the primary documents in the larger study for coding by an external rater. This sample was compared with the codes assigned by the researcher. There was an $80 \%$ level of agreement in the coding of the text by the two raters. The focus groups were conducted in both contexts, and contributed similar information without any new revelations, which was considered as data saturation. At the conclusion of each focus group, member-checking was done by presenting a summary of each focus group to the participants for their approval immediately following the session. This measure deviated from conventional member checking (which is usually done at the conclusion of a study), to limit costs and time required by an additional contact session. Field notes by the researcher further supplemented the summary and transcription of the audio-recording.

At the completion of the workshop the researcher compared notes with the assistant moderator (who was one of the district facilitators) and reflected on the procedure, the participation, as well as the outcomes of the session. The researcher further reflected on the focus group shortly afterwards. The transcriptions were done according to literature guidelines. In order to protect the identity of the participants numbers were assigned to each, and they were referred to as T1, T2, etc. Credibility was increased by including verbatim quotations that were provided as data and an audit trial.

\section{Data analyses}

In this qualitative research content analyses were used as they provided clear descriptions of how the participants implemented the strategies in their classrooms and also documented their experiences. The qualitative data were analysed with ATLAS-ti (Thomas Muir Scientific Software Development, 2003-2004) (qualitative descriptive analysis), which allowed for enumeration. The counting of the codes enabled the researcher to determine the prominence of the various categories. Tabulations were used to interpret the data as they reflected how the strategies were implemented, and how the support was perceived.

To further explore the significance of the findings the qualitative data (codes) were converted to quantitative data by reducing them to numerical information in three dichotomous categories $(0-$ neutral/ nonspecific, 1 - negative, or 2 - positive). The quantified qualitative data were summarised and presented on theme level, category level, and in specific cases also on a code level. Final conclusions were drawn once a validation process confirmed the interpretation of the inferences.

\section{Results and discussion}

\section{Implementation of the strategies in the classroom}

Based on the responses to the questionnaires, reflections and focus groups, the following information was obtained on the participants' implementation of strategies to facilitate numeracy.

\section{Use of terminology}

The results showed that of the items coded, 53\% ( $n=15)$ confirmed the acquisition of new terminology after the workshop. Some participants demonstrated better recall than others, which may be the result of more prior knowledge, which became evident from the following quote:

T: I'm thinking about the one-to-one correspondence, and the seriation, classification. That is what they are doing. So when they come to Gr 1 we expect them to know those things.

The use of new terminology was, however, not generalised during the training as became evident when $64 \%(n=14)$ of the items were coded as 'inability to recall the information'. The inability to recall the terminology may have been related to some participants having limited language proficiency in English as is evident from the following example:

T: I am talking about ... I forgot the thing that you showed us. The ... the ... when you taught the kids the heavy, heavier?

A.M: The scale?

T: The scale. Yeah!

When participants could not recall the correct terminology, they described the concepts in their own words. This relates to the 'awareness level' of knowledge acquisition, which is one level higher than the entry level in knowledge acquisition (Miller \& Watts, 1990). In this case the participants were aware of the information, but in several instances their knowledge was not applied in their classrooms. It is possible that some of the participants could not recall the terminology because they had not completed their portfolio assignments.

The participants reported that although the learners understood the language used in the classroom (e.g. when money was referred to as 'five-bob'), they became confused when standard terminology was used ('five rand') on their worksheets, which were mostly in English. One participant described the situation as follows:

Primary document (PD) 5, line 76, focus group 2, in 2005: 'They know the money when we talk [in]formally but when write(sic),.... oh chaos!'

It may be confusing for a learner when the teacher states a problem in one way while the text presents the same problem in a different manner with different vocabulary and terminology. Learners from disadvantaged communities may not have had access to books or experiences that would allow them to develop appropriate concepts and terminology for numeracy. The use of incorrect terminology may cause learners to experience difficulty in standardised assessment procedures (e.g. the GDE's annual numeracy challenge, or the Third International Mathematics and Science Study (TIMSS)), as the formal terminology may be unfamiliar to them. It is important that teachers consistently provide accurate examples of numeracy terminology and vocabulary (Thompson \& Rubinstein, 2000), and participants therefore need to be alerted to the consequences of not doing so.

\section{Prior knowledge of teachers}

The conceptual knowledge for teaching numeracy is as much about pedagogy as it is about content. Some of the participants reported that they had never before addressed specific numeracy concepts in class (e.g. the concepts of estimation or three-dimensionality), because they themselves had a limited understanding of the concepts.

Diary entry 15 on pilot workshop 3: In one focus group it was determined that the participants had never before addressed the term 'estimate' (which is required by the NCS), because the term was unfamiliar to them.

This is attributed to the fact that not all the participants had similar qualifications or displayed similar levels of competence when they entered the programme, and therefore they differed in their understanding of the material during the programme. The inability to explain certain terminology may be ascribed to limited prior knowledge and/or inadequate English language proficiency. The national curriculum statement (NCS) is available in English, however, the vocabulary used and concepts referred to were not understood by all the participants. Limited conceptual knowledge of teachers causes poor performance of learners, and therefore indicates a need for continued teacher support.

\section{Expectations of learners}

Reflective notes of the researcher after marking the portfolio assignments indicated that some participants applied inappropriate activities that appeared to be more suitable for lower grades than for the specific grade levels that they were teaching. 
Line 28, reflection of the trainer: From the assignments, it is clear that in many cases the teachers provided numeracy activities which seemed more suitable for lower grades than for the specific grade level (do they have low expectations?))

In addition, it was found that the teaching materials used often did not meet the level of learning required, which is consistent with results obtained by Thusi (2006). Such materials are unlikely to develop higher order thinking skills in their learners. The results show that some of the participants underestimated the learners' abilities, or had too-low expectations. It is also possible that the learners were too far behind in the curriculum to meet the standards set for specific grade levels.

Low teacher expectations of learners' achievement in low-income communities are well documented. The Reeves study (1998) of teaching and learning Gr 4 mathematics, as well as recent reports from the Khanyisa project (Khoza, 2007), found that teachers had fairly low expectations of their learners as a whole as tasks were not cognitively demanding, which may have been the case in this context. In addition, it is also known that learners from poor SES mostly have limited or no preschool experience, which places them at risk of failing (Botha et al., 2005:697). It is therefore possible that these learners required more time to catch up.

\section{Language issues}

\section{Resources in the LoLT}

Pluddemann, Mati and Mahlalela-Thusi (1998) reported that teachers favoured the use of English materials, which was also confirmed by this study. Many of the participants made use of worksheets which they copied from English workbooks. Because of a lack of suitable resources in the LoLT, many teachers bought a numeracy workbook written in English from the local news agency, and photocopied the worksheets for their learners. English worksheets were used despite the school's policy on the LoLT, because the teachers had no other resources available. English is an additional language for all the learners in these particular schools and this practice could have affected their acquisition of the numeracy concepts taught in the classrooms. When considering that cognitive academic language takes 5 - 7 years to develop (Cummins, 2000), the use of English workbooks could have implications for the quality of education in this context. Ideally, basic concepts should first be acquired in the mother tongue, and even though workbooks were available in Northern Sotho (e.g. Oxford University Press), schools in these specific contexts did not have access to them or the funds to buy them. In addition, it was noted by the researcher that:

Line 8, reflection of the trainer: Even though workbooks could be provided in the LoLT, it would not necessarily meet the diverse needs of all learners.

Such practices indicate a need for teacher support in implementing the numeracy curriculum.

\section{Language use in the classroom}

Despite their schools policy in terms of the LoLT, some of the participants were also confused as to whether they should not rather use English when teaching numeracy because it was much easier for them to use the English terminology and to have access to English worksheets. The use of English terminology and vocabulary in these contexts was not uncommon, which makes it imperative to use code switching to an African language when introducing new concepts in numeracy (Du Plessis, 2005). The importance of code switching therefore needs to be emphasised in future CPD programmes. Some of the participants discovered the importance of L1 to develop 'numeracy' skills as illustrated in the following comment:

Line 49, focus group on workshop (WS) 3: T1: 'He must understand the language first.'

They also learnt how to facilitate numeracy skills in a constructive manner (by making use of real objects and live experiences, and using the correct numeracy vocabulary and terminology), as was confirmed by $89 \%$ of items coded $(n=35)$.

\section{Importance of play and culture in learning}

The participants' portfolios contained evidence that the language of numeracy was facilitated by use of indigenous games (e.g. 'Morabaraba', a board game usually played with stones, which requires counting), stories, songs, and teaching resources. According to Arvedson (2002) children learn the conventional count words of their culture and use these words to manipulate larger and larger sets of objects and events. A child's count range is evidence of the child's opportunities to experience and manipulate the conventional count words of that child's culture (Arvedson, 2002). The use of indigenous games by the participants provided a natural experience for the facilitation of the learners' numeracy development. The importance of culture in teaching and learning became evident in the following quote from the researcher's field notes:

Line 97, reflection on focus group 1, semi-rural context: One of the participants expressed sadness because her own son attended a school with English as LoLT, which caused him to lose his language and culture.

\section{The value of the CPD programme to the participants}

There was convincing evidence that the CPD programme was valued by the participants.

\section{Impact on creativity}

A few participants experienced themselves as becoming more 'creative and innovative' ( $89 \%, n=9)$ as they had acquired new 'strategies' and generated new ideas to implement in class $(n=28)$.

PD11, line 101, focus group 3: T2: But at the course, we got those ideas. I got the polystyrene. Then the shapes, when I drew this, it were one dimension. The moment I had it on polystyrene it was three dimensions! So the HOD and I, we went to the rubbish heap, and got that polystyrene.

The participants testified that the workshop facilitated their understanding of three-dimensionality, which confirms on poor content knowledge prior to the training. Sufficient content knowledge enables teachers to employ inventive and creative opportunities for learning (Van der Sandt \& Nieuwoudt, 2005). Such creativity was described by Spady (2001: 34) as one of the common threads of quality learning because '...learning is not just absorbing content from printed material; it's an inherent part of living simply because living is a continuously unfolding array of new input and experiences.'

\section{Impact on functioning}

Several participants (who previously relied heavily on 'commercial programmes' to teach) reported that the training helped them to become more independent from using such programmes and also more confident in creating their own materials and activities.

Line 235, pilot focus group 1: In Gr R ... and another thing - the workshop also help us a lot - to be creative. They thought in our language we can only teach one, two, three. Now we can create our own stories, our own riddles, and our own songs.

The ability to develop their own lesson plans increased the participants' confidence, which in turn can be related to improved learner achievement. The benefit of this workshop to the participants was further confirmed by an external evaluation of a group of learning support teachers (LSE) from the GDE, who unofficially attended the workshop in the urban context. They viewed the information taught in the workshop as having the potential to change the manner in which teachers teach numeracy and thought it would be valuable in their own support of teachers and learners who experience challenges in numeracy.

Line 24, testimonials from teacher support teachers: The teachers approach is going to be different especially with numeracy.

Line 12, testimonials from teacher support teachers: I have developed competence and skill in teaching numeracy in the foundation phase because I have no teaching experience of this phase. And will be able to address the problem of LoLT at English medium schools.

Some of the participants were not specifically qualified to teach the foundation phase and were grateful for the opportunity to learn practical skills for supporting young learners with numeracy. They 
may not have been fully equipped to deal with the demands of foundation-phase teaching and its specific curriculum, which in itself may also be a factor to be considered in providing additional support of teachers.

\section{Limitations of the study}

Several challenges were experienced during the research. The data showed a level of non-response in the open-ended questions in the questionnaires, as well as in the critical reflections in the portfolio assessments. This could be attributed to the participants not being familiar with reflective practices, but also to the use of language, literacy levels, and aspects related to timing, as well as logistics of the programme. The threat of subjectivity is acknowledged as the first author was involved with these communities over a prolonged period of time, and personally conducted all aspects of the research. Subjectivity is considered as one of the weaknesses of qualitative research but by paying close attention to credibility and using a reflection diary, personal bias was limited.

\section{Conclusions and implications}

Based on the findings of the literature review it is clear that the SLP has a crucial role to play in the support of teachers regarding numeracy. This CPD programme proved to be an effective method to make teachers aware of the importance of language in the acquisition of numeracy skills and how to facilitate the process. The programme was valued by the participants as it allowed them to address specific assessment standards that they were not able to do prior to training and to gain confidence.

Within a collaborative approach to teacher support, SLPs need to provide workshops where teachers can see how numeracy concepts are being demonstrated by using the correct use of language. Such workshops would also allow teachers to practise strategies in role play, which is non-threatening. In turn, teachers, as mediators of learning, need to create multiple opportunities for learners to practise the correct use of numeracy language (e.g. through general as well as small-group discussions).

It cannot be taken for granted that teachers themselves understand the vocabulary in the curriculum, which implies that SLPs need to include explanations and demonstrations of the concepts in a support programme. Such support should not be restricted to classroom teachers only, but needs to include the learning support teachers who are responsible for learners at risk of failure, as well as the district facilitators. In cases where teachers use English as LoLT, the use of code switching should be emphasised when explaining numeracy concepts. SLPs working in schools with limited resources should support teachers in the translation of English worksheets to the LoLT. Although the Department of Education is currently committed to supplying the necessary resources, school leadership should be encouraged to plan ahead and order their supplies in time.

From the results obtained from the larger study, it was determined that CPD activities are required on an ongoing basis because of diversity of teachers (e.g. qualifications, language proficiency). Workshops will be more effective if conducted with smaller groups as opposed to large-group workshops. Ideally, teachers from a particular cluster or school will learn best by sitting around a table for shorter topic-specific sessions, which are offered over a prolonged period of time. The present study provides evidence that the support of teachers through a CPD programme was effective and was perceived as being positive by the participants. SLPs working in the school system who expand their role to support teachers through CPD programmes, can contribute to ensuring educational relevance in teaching and learning and to the implementation of the curriculum, which in turn fulfils some of the critical roles of SLPs working in school contexts.

Acknowledgements. The research was funded by a grant from the Shuttleworth Foundation.

\section{References}

Arvedson, P. J. (2002). Young children with specific language impairment and their numerical cognition. Journal of Speech, Language and Hearing Research, 45, 870-982. doi:10.1044/1092-4388(2002/079)

American Speech-language Hearing Association (ASHA). (2010). Roles and responsibilities of speech-language pathologists in schools: Rockville, MD: ASHA.

Barwell, R., Leungh, C., Morgan, C., \& Street, B. (2005). Special issue: Language and maths. Language and Education 19(2): 118-126

Baxen, J., \& Green, L. (1999). Primary teachers' use of learning materials. In N. Taylor \& P. Vinjevold (Eds.) Getting learning right: Report of the president's education initiative research project (pp. 261-267). Johannesburg. Department of Education and the Joint Education Trust.

Botha, M., Maree, J. G., \& de Witt, M. W. (2005). Developing and piloting the planning for facilitating mathematical processes and strategies for preschool learners. Early childhood development and care, 175(7\&8), 697-717. doi:10.1080/0300443042000302582

Brennan, M. K. (2008). Mathematics from a language perspective. Paper presented at the ASHA Convention: 'Winds of Change'. Retrieved from www.asha.org/Publications/leader/2008/080715/080715d.htm

Cummins, J. (2000). Language, power, and pedagogy: Bilingual children in the crossfire. Clevedon: Multilingual Matters. Department of Basic Education. (2011). Report on the 2008-2009 annual surveys for ordinary schools. Pretoria: Basic Education. Department of Education. (2002). Revised National Curriculum Statement for Schools: Grades R-9.

Du Plessis, S. (2005). Multilingual preschool learners: A collaborative approach to communication intervention University of Pretoria, Pretoria.

Du Toit, P., Froneman, D., \& Maree, K. (2002). Mathematics learning in the foundation phase: facilitating a parentteacher partnership. Acta Academica, 34 (2), 154-181.

Elkind, D. (2001). Much too early. Education Next, 12, 8-14.

Gawned, S. (1993). An emerging model of the language of mathematics. In J. Bickmore-Brand (Ed.), The language of mathematics (pp. 27-41). Portsmouth, NH: Heinekenn.

Harrison, L. J., McLeod, S., Berthelsen, D., \& Walker, S. (2009). Literacy, numeracy, and learning in school-aged children identified as having speech and language impairment in early childhood. International Journal of Speech-Language Pathology, 11(5), 392-403. doi:10.1080/17549500903093749

Kathard, H., Ramma, L., Pascoe, M., Jordaan, H., Moonsamy, S., Wium, A. M., du Plessis, S., Pottas, L., \& Banu Khan, N. (2011). How can speech language therapists and audiologists enhance language and literacy outcomes in South Africa? (And why we urgently need to)". South African Journal of Communication Disorders, 53 (Special edition): 1-8. Khoza, G. (December 2007). A model for improving schooling. Jet Bulletin, 1-12.

Koponen, T., Mononen, R., Rasanen, P., \& Ahonen, T. (2006). Basic numeracy in children with specific language impairment: heterogeneity and connections to language. Journal of Speech, Language, and Hearing Research, 49, 58-73. doi:10.1044/1092-4388(2006/005)

Mercer, N., \& Sams, C. (2006). Teaching children how to use language to solve maths problems. Language and Education, 20 (6): 507-528. doi10.2167/le678.0

Miller, A., \& Watts, P. (1990). Planning and managing effective professional development. Essex: Longman.

Monroe, E., \& Omre, M. (2002). Developing mathematical vocabulary. Preventing School Failure, 46 (3). doi:10.1080/10459880209603359

Pluddemann, P., Mati, X. \& Mahlalela-Thusi, B. (1998), Problems and possibilities in multilingual classrooms in the Eastern Cape. In N. Taylor \& P. Vinjevold (Eds.), Getting learning right: Report of the president's education initiative project (pp. 317-319). Johannesburg: Joint Education Trust/Department of Education.

Raiker, A. (2002). Spoken language and mathematics. Cambridge Journal of Education, 32(1), 45-61. doi:10.1080/03057640220116427

Reeves, C., \& Long, C. (1998). An investigation into grade 4 mathematics teaching and learning. In N. Taylor \& P. Vinjevold (Eds.), Getting learning right: Report of the president's education initiative research project (pp. 323324). Johannesburg: Joint Education Trust/Department of Education.

Reeves, N. (1993). The mathematics-language connection. In J. Bickmore-Brand (Ed.), Language in mathematics (pp. 90-99). Portsmouth, NH: Heineken.

Samuelson, V. (2008). The language of math: What is the SLP's role? Paper presented at the ASHA Convention 'The Winds of Change'. Retrieved from www.asha.org/Publications/leader/2008/080715/080715d.htm

Schleppegrell, M. (2007). The linguistic challenges of mathematics teaching and learning: A research review. Reading and Writing Quarterly, 23, 139-159. doi: 10.1080/10573560601158461

Setati, M. (1999). Innovative language practices in multilingual mathematics classrooms. In N. Taylor \& P. Vinjevold (Eds.), Getting learning right: Report of the president's education initiative research project. Johannesburg: Join Education Trust/Department of Education.

Spady, W. G. (2001). Beyond counterfeit reforms: Forging an authentic future for all learners. Lanham, MD: Scarecrow Press Thomas Muir Scientific Software Development. (2003-2004). ATLAS-ti: The knowledge workbench V5.0. Berlin Thomas Muir Scientific Software Development.

Thompson, D. R., \& Rubinstein, R. N. (2000). Learning mathematics vocabulary: Potential pitfalls and instruction strategies. Mathematics Teacher, 93(7), 568.

Thusi, L. B. (2006). The implementation of outcomes based education in township primary schools. University of Johannesburg, Johannesburg.

Torbeyns, J., van den Noortgate, W. \& Ghesquirer, P. (2002). Development of early numeracy in 5-7 year old children: A comparison between Flanders and the Netherlands. Educational Research and Evaluation, 8(3), 249-275. do: 10.1076/edre.8.3.249.3855

Vacca, A., \& Vacca, R. (1996). Content area reading (5th ed.). New York: Harper Collins.

Van der Sandt, S., \& Nieuwoudt, H. D. (2005). Geometry content knowledge: Is pre-service training making a difference? African Journal of Research in SMT Education, 9(2), 109-120.

Wium, A. M., Louw, B., \& Eloff, I. (2010). Speech-language therapists supporting foundation phase teachers with literacy and numeracy in a rural and township context. South African Journal of Communication Disorders 57(1), 14-22. 


\section{Appendix A. CPD: Supporting teachers with a three-pronged approach The continued professional programme (CPD) consisted of a three-pronged approach (Wium et al., 2010): Table A1. A three-pronged approach used in the CPD programme}

Component

'Language in numeracy'
Practical component

Mentoring component

\section{Procedure}

Workshops have been identified as important 'confidence boosters' (Baxen \& Green, 1999: 264). Considering that confidence is an important component of competence, this CPD programme offered a full-day workshop in two contexts (semi-rural and urban),

The aims of this workshop were to alert teachers to the importance of language use in numeracy, and to empower them to facilitate the acquisition of the language required for numeracy development. The workshop was conducted as follows:

- The workshop firstly emphasised the importance of language in numeracy and explained the various discourses in the classroom, using PowerPoint (PPT) and whole-class discussion.

- The workshop facilitator demonstrated to the delegates how to explain various concepts with accompanying instructional vocabulary, by using real objects/activities and items to manipulate (e.g. containers with water to demonstrate volume, a scale for weighing and to demonstrate mass, bottle caps for sorting, counting, etc).

- Four participants were grouped in grade levels and were each handed four different written problems cut from numeracy worksheets (which were obtained from grade level numeracy workbooks) (e.g. Oxford Books).

- The groups were required to use a role play scenario to explain and clarify the specific vocabulary that typically occurs in the curriculum.

- The groups presented their scenarios to the entire class, which was a fun manner to learn from each other. The role play activities provided the participants with the opportunity to first observe the strategies before they were required to apply the strategies over a period of time and to reflect on the process.

Following the workshops the participants were required to apply their knowledge in their classrooms for a period of 3 - 4 weeks, which was documented in a portfolio.

The mentoring component consisted of collaborative peer learning and feedback on portfolio assignments (Wium et al., 2010). The portfolio consisted of lesson plans for each week of the implementation period, monitoring sheets which were completed to monitor the participation of three learners from varying performance levels, selfreflection sheets, and artefacts used in their lessons. The workshop facilitator provided feedback on the lesson plans and the portfolios.

\section{Appendix B. Vocabulary items in the foundation-phase curriculum}

Table B1. Vocabulary items in the foundation-phase curriculum for English first language (Department of Education, 2002)

\begin{tabular}{|c|c|c|}
\hline \multicolumn{3}{|l|}{ Gr R and Gr 1} \\
\hline Add & Count & Join \\
\hline Arrange & Cross & Link \\
\hline Build & Double & Match \\
\hline Circle & Fill in & Tick \\
\hline Colour & Find & Trace \\
\hline Compare & Finish & Use \\
\hline Complete & Fold & Work out \\
\hline Copy & Halve & \\
\hline \multicolumn{3}{|l|}{ Gr 2 and 3} \\
\hline Above/up & Between & Estimate \\
\hline Add, addition & Bigger & Evaluate \\
\hline After & Complete & Far, near \\
\hline All capacity $(1, \mathrm{ml}, \mathrm{kl})$ & Complete the equation & First, second, third, etc. \\
\hline Measurement $(\mathrm{cm}, \mathrm{mm}, 10 \mathrm{~mm}=1 \mathrm{~cm})$ & Decrease & Full \\
\hline Apart & Different & Halve \\
\hline Ascending order & Discuss & Higher/lower \\
\hline Before & Divide & How many? \\
\hline Below, down & Division & Increase \\
\hline \multirow[t]{3}{*}{ Beside } & Double & Less than \\
\hline & Empty & Long time \\
\hline & Equal to & \\
\hline
\end{tabular}




\section{Appendix C. Discourses used in the classroom \\ Table C1. Levels 3 and 4 of discourses used in the classroom (Gawned, 1993)}

Level 3: The language required for numeracy

\section{Language of reasoning (problem solving)}

The language of reasoning is used by teachers and learners in problem-solving contexts and includes complex sentences used for inferences, justifications, comparisons and predictions. This type of language is determined by the discourse used for description, comparison, and reflection.

\section{Language of the numeracy curriculum}

- Language of the numeracy curriculum (refer to Appendix B, Table B1) includes terminology which has to be explicitly taught and learnt (Botha et al., 2005), as it is essential for developing higher level thinking skills such as analysis, discussion, problem solving and design in relation to the subject matter.

- Learning of the terminology of the mathematics curriculum requires that learners firstly develop an understanding of the underlying concepts through their own experiences, problem-solving solutions, and strategies (Du Toit, Froneman \& Maree, 2002).

\section{Language of activities}

Classroom activities require both descriptive language and procedural language (Gawned, 1993).

- Descriptive language: Allows the user to participate in an activity (e.g. labels, attribute terms and noun phrase constructions to discuss relationships between numbers, concepts, etc.),

- Procedural language: Used to explain how procedures need to be conducted and provide reasons for classifying or grouping items in a particular manner.

\section{Language of mathematics literacy}

The language of mathematics literacy:

- Develops once learners start with the mathematics curriculum (not applicable to the numeracy curriculum in the foundation phase).

- Refers to the representation and recording of mathematics (e.g. graph construction, diagramming, mapping, writing the digits accurately, etc.) (Gawned, 1993)

- Brennan (2008) distinguishes between 'Ordinary English (OE), Math English (ME) and Specialised Meanings (SM)'.

- OE refers to the same word that has the same meaning,

- ME has only a primary meaning in mathematics, and is taught by the teacher (e.g. 'numerator'). It becomes a language in its own right.

- SM: the same word has different meanings (e.g. 'average', 'range,' 'equal', 'divide', etc.), which may or may not necessarily be taught by the teacher.

\section{Level 4: Construction of meaning (Gawned, 1993)}

Teachers need to ensure that learners acquire the necessary vocabulary and language competence to enable them to understand the mathematical concepts being taught.

Learners with a well-developed vocabulary can devote all their attention to the new concepts and the next step and do not experience difficulties in understanding the meaning of the words used.
Methods of facilitation

Teachers need to:

- Use discussion to clarify meaning and help learners to absorb terminology and understand the concepts (Department of Education, 2002).

- Create opportunities for talking about learners' ideas in relation to their experiences.

- Create an awareness of thought, e.g. to encourage, predict, and hypothesise, as well as to create opportunities in terms of questions and situations for the use of 'if/then', 'what if?', 'why?', 'what would happen?', 'what did happen?' and 'how did you know?' (Reeves, 1993: 91).

- Firstly make use of manipulates, shapes, and collections of objects in play activities (Botha et al., 2005).

- When translation of mathematical terms to an African language is not possible, an English word can be used, but should also be described in the African language. Be aware of possible ambiguity in word meaning, and actively clarify and teach unfamiliar terms.

- Avoid using English workbooks in classes where the LoLT is an indigenous language because the grammatical and morphological structures differ.

- Start teaching of terminology and vocabulary for numeracy when the learner is being taught the vocabulary necessary to start reading.

Learners need to be encouraged to talk about procedures when working in groups and to engage actively with real objects in a constructive manner to develop an understanding of the concepts.

Teachers should use clear syntax in order to allow learners to understand

what is required of them in completing the computation.

- Brennan (2008) suggests avoidance of long, dense noun phrases, and complex meanings should be unpacked.

- Numerical comparisons should be practised (e.g. 'write a sentence that gives the same information as the information in the diagram').

- Teachers need to match the sentence structures used for writing mathematical problems (e.g. in word sums) with the learners' levels of comprehension. Accordingly, learners' written language needs to be practised in the classroom.

\section{Methods of facilitation}

Pay attention to conceptual confusion when everyday metaphors are used in the classroom. Teachers should be aware of cause for confusion, e.g.

- Pronoun ambiguity: (e.g. clarify who does 'we' refer to when explaining calculations - e.g. "we take 10 from the 10 s column").

- When teachers make the shift from 'I' to 'you' it implies generalisation.

- The use of passive voice (e.g. 'the answer is written below the line') where agency becomes suppressed and changing from human agency to disciplinary agency.

Allow more opportunity for dialogue about these concepts and processes and encourage learners to apply them to their lives in small groups. 